Pacific Journal of Mathematics

FIRST INTEGRALS FOR A DIRECTION FIELD ON A SIMPLY 


\title{
FIRST INTEGRALS FOR A DIRECTION FIELD ON A SIMPLY CONNECTED PLANE DOMAIN
}

\author{
JOEL L. WEINER
}

Let $\mathscr{D}$ be a simply connected plane domain and let $r$ be a positive integer or $\infty$. By a $C^{r}$ direction field $\Phi$ on $\mathscr{D}$ we mean $C^{r}$ mapping $\Phi$ : $\mathscr{D} \rightarrow G_{2,1}$, the projective line consisting of lines through the origin, 0 , in the plane. A $C^{r}$ first integral of $\Phi$ is a $C^{r}$ function $f: \mathscr{D} \rightarrow \mathbf{R}$ such that each level set of $f$ has no interior and is a union of members of the family, $\mathscr{F}$, of maximal integral curves of $\Phi$. We show, in general, that first integrals do not exist and then give a necessary and sufficient condition for a $C^{r}$ first integral to exist. When $\Phi$ has a first integral we also show that there exists a local diffeomorphism $\mu: \mathscr{D} \rightarrow \mathbf{R}^{2}$ such that $\Phi$ is mapped by $\mu$ into the (constant) vertical direction field on $\mu(\mathscr{D})$.

Introduction. W. Kaplan [4] has shown that a $C^{r}$ direction field $\Phi$ has a $C^{0}$ first integral; more precisely, he shows there exists a continuous function $f: \mathscr{D} \rightarrow \mathbf{R}$ such that

(1) for every $c \in \mathbf{R}, f^{-1}(c)$ consists of at most countably many curves of $\mathscr{F}$, and

(2) in every neighborhood of a point $P_{0} \in \mathscr{D}$ there are points $P$ for which $f(P)>f\left(P_{0}\right)$ and points $P$ for which $f(P)<f\left(P_{0}\right)$.

E. Kamke [3] and R. Finn [1] showed the existence of a $C^{r}$ first integral for a simply connected relatively compact domain $\mathscr{D}$, assuming, roughly, that $\Phi$ has a $C^{r}$ extension to the closure $\overline{\mathscr{D}}$; the Kamke $\Phi$ should be $C^{r}$ on a domain containing $\overline{\mathscr{D}}$, while for Finn the boundary of $\mathscr{D}$ should be $C^{r}$. Finn, in fact, establishes necessary and sufficient conditions for the existence of first integrals in multiply connected domains and obtains conditions that apply to singular situations.

Since, as is well known, all simply connected domains in the plane are diffeomorphic, we will take $\mathscr{D}$ to be the plane, $\mathbf{R}^{2}$, throughout. However, for specific applications and examples it is often convenient to use other models. Thus we are led to the following question. If $\Phi$ is $C^{r}$ on $\mathbf{R}^{2}$, does there exist a globally defined $C^{r}$ first integral of $\Phi$ without imposing additional restrictions on $\Phi$ ? In $\S 1$ we show that additional conditions must be imposed. This is a consequence of the following theorem. 
THEOREM 1. There exists on $\mathbf{R}^{2}$ a $C^{\infty}$ direction field $\Phi$ for which the following holds: there is an open set $U \subset \mathbf{R}^{2}$ such that if $f: \mathbf{R}^{2} \rightarrow \mathbf{R}$ is $C^{1}$ and constant on the integral curves of $\Phi$ then $f$ is constant on $U$.

The only obstacle to obtaining a $C^{r}$ first integral for a $C^{r}$ direction field defined on a simply connected plane domain is illustrated in a construction that appears in the proof of Proposition 1. It is the repeated use of this obstacle that is employed to obtain the result mentioned in Theorem 1.

In $\S 2$ we define what we mean by an $r$-normal direction field $\Phi$ when $\Phi$ is $C^{r}$. Then Theorem 2 states that $\Phi$ being $r$-normal is necessary and sufficient for $\Phi$ to possess a $C^{r}$ first integral whose differential does not vanish on $\mathbf{R}^{2}$. We then proceed to show the following:

Theorem 3. Let $\Phi$ be a $C^{r}$ directional field on $\mathbf{R}^{2}$. Then $\Phi$ is a $r$-normal if and only if there exists a $C^{r}$ local diffeomorphism $\mu: \mathbf{R}^{2} \rightarrow \mathbf{R}^{2}$ such that $d \mu(\Phi)$ is the (constant) vertical direction field.

Theorem 3 is much closer in spirit to the results of E. Kamke and R. Finn mentioned above. We also indicate in $\$ 2$ that $\mu$ of Theorem 3, in general, cannot be a diffeomorphism. We close $\$ 2$ by considering a pair of independent direction fields on $\mathbf{R}^{2}$. Theorem 4 is a "uniformization" theorem for a pair of independent direction fields.

Finally, $\$ 3$ contains applications of our results to the theory of differential geometry and to the normalization of linear partial differential equations.

After completion of this work, it was found that T. Wazewski [6] has an example of a simply connected domain and a direction field such that any first integral is constant, thus anticipating Theorem 1 of this paper. However, Wazewski's example is much more complicated than ours.

1. Counterexample. Before we proceed to the counterexamples, let's first precisely define what we mean by a first integral.

Definition 1. Let $\Phi$ be a $C^{r}$ direction field on $\mathbf{R}^{2}$. We say that the $C^{s}$ function $f: \mathbf{R}^{2} \rightarrow \mathbf{R}(s \leq r)$ is a $C^{s}$ first integral of $\Phi$ in the strong sense if

(1) $f$ is constant on members of $\mathscr{F}$, and

(2) $d f$ vanishes nowhere in $\mathbf{R}^{2}$.

Proposition 1. There exist $C^{\infty}$ direction fields $\Phi$ on $\mathbf{R}^{2}$ which do not have any $C^{1}$ first integrals in the strong sense. 
Proof. We will construct an example on a suitable domain $\mathscr{D}$ instead of the plane. Let $b: \mathbf{R} \rightarrow \mathbf{R}$ be defined such that

(1) $b$ is $C^{\infty}$,

(2) $b(x)=1$, for $x \leq-1 / 2$, and

(3) $b(x)=0$, for $x \geq 1 / 2$.

Also, let $g:[0, \infty) \rightarrow[0, \infty)$ be defined such that

(1) $g$ is $C^{0}$ and $g(0)=0$, and

(2) $g$ is $C^{\infty}$ on $(0, \infty)$ and $g^{\prime}(x)>0$ for $x>0$.

Denote the open upper half-plane by $\mathscr{H}$ and define a transformation $\tau$ : $\mathscr{H} \rightarrow \mathscr{H}$ by

$$
(u, v)=\tau(x, c)=(x, c b(x)+g(c)(1-b(x))) .
$$

Clearly $\tau$ is a $C^{\infty}$ diffeomorphism. Therefore, the tangent directions to the curves $x \rightarrow \tau(x, c)$ determines a $C^{\infty}$ direction field which is denoted by $\phi$. Let $\mathscr{D}$ be the complement of $\{(u, v) \mid-1 \leq u \leq 1$ and $v \leq 0\}$. Note that $\phi$ is horizontal for $u \leq-1 / 2$ and $u \geq 1 / 2$; consequently we can extend $\phi$ to a $C^{\infty}$ direction field $\Phi$ on $\mathscr{D}$ by requiring that $\Phi$ be horizontal where $v \leq 0$ on $\mathscr{D}$. See Figure 1 which displays $\mathscr{D}$ and the integral curves of $\Phi$. Let $g^{\prime}(0+)$ denote the derivative of $g$ at 0 from the right. Clearly there exist $g$ satisfying (1) and (2) above as well as $g^{\prime}(0+)=0$.

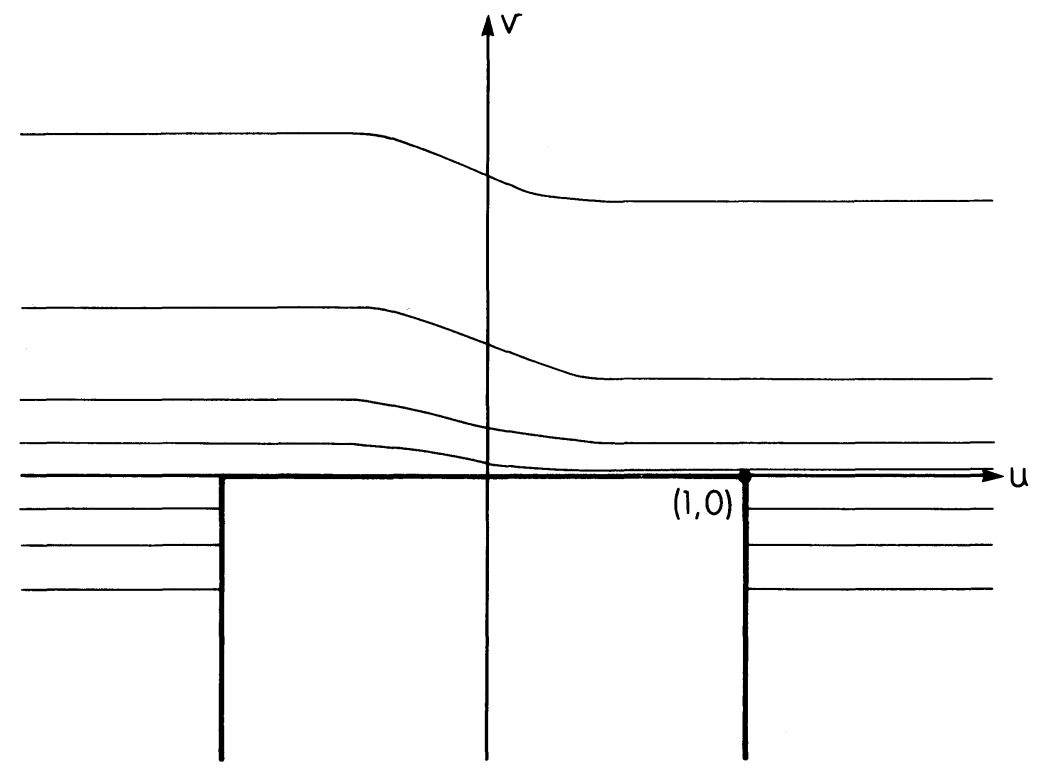


Let $f$ be a $C^{1}$ function on $\mathscr{D}$ which is constant on the integral curves of $\Phi$. We will show that if $g$ has the properties just prescribed then $d f_{(u, v)}=0$ for $v=0$ and $u<-1$. For any $u<-1$ we have $f(u, t)=$ $f(2, g(t))$, for $t \geq 0$, by construction. Consequently,

$$
\frac{\partial f}{\partial t}(u, 0)=\frac{\partial f}{\partial t}(2,0) \cdot g^{\prime}(0+)=0 .
$$

Since $f(x, 0)$ is constant for $x$ near $u$, we have $d f_{(u, 0)}=0$.

REMARK. The example presented in this proof was inspired by an observation of Hans Samelson.

In the light of Proposition 1, one could ask for a weaker definition of the first integral of $\Phi$.

Definition 2. Let $I$ be an interval of real numbers. A $C^{1}$ curve $\Upsilon$ : $I \rightarrow \mathbf{R}^{2}$ is said to be transversal to $\Phi$ if $\Upsilon^{\prime}(t) \notin \Phi(\Upsilon(t))$, for all $t \in I$. A $C^{s}$ function $f: \mathbf{R}^{2} \rightarrow \mathbf{R}$ is said to be a $C^{s}$ first integral of $\Phi$ in the weak sense if

(1) $f$ is constant on members of $\mathscr{F}$, and

(2) $f$ is strictly monotonic on all curves transversal to $\Phi$.

However, this or perhaps even a weaker definition will not allow for the existence of a $C^{r}$ first integral in all cases where $\Phi$ is a $C^{r}$ direction field on $\mathbf{R}^{2}$.

THEOREM 1. There exists on $\mathbf{R}^{2}$ a $C^{\infty}$ direction field $\Phi$ for which the following holds: there is an open set $U \subset \mathbf{R}^{2}$ such that if $f: \mathbf{R}^{2} \rightarrow \mathbf{R}$ is $C^{1}$ and constant on integral curves of $\Phi$ then $f$ is constant on $U$.

Proof. We will construct such a field on a suitable domain $\mathscr{D}$ rather than on $\mathbf{R}^{2}$. The idea behind the example is to use repeatedly the construction in the proof of Proposition 1 to obtain in $U$ a dense subset on which $d f=0$; then by continuity $d f=0$ on $U$.

The domain $\mathscr{D}$ to be constructed will be contained in $(-\infty, \infty) \times(0,1)$ and $\{0\} \times(0,1) \subset \mathscr{D}$. The field $\Phi$ is constructed so that

(i) if $f$ is $C^{1}$ and constant on members of $\mathscr{F}$, then $d f_{(0, y)}=0$, where $y$ is a dyadic between 0 and 1 .

By continuity $d f_{(0, y)}=0$ for $0<y<1$. Hence $f$ is constant on $\{0\} \times(0,1)$. Moreover, $\Phi$ is constructed so that

(ii) if a member of $\mathscr{F}$ meets $\{0\} \times(0,1)$, then it meets $\{0\} \times(0,1)$ transversally. 
Consequently there is a neighborhood, $U$, of $\{0\} \times(0,1)$ on which $f$ must be constant and this neighborhood may be chosen independently of $f$. We now proceed to construct the field $\Phi$ satisfying properties (i) and (ii).

Let $\Phi_{0}$ be the standard horizontal direction field on $\mathscr{D}_{0}=(-\infty, \infty) \times$ $(0,1)$. Modify $\Phi_{0}$ on $x>0$ to obtain a field $\Phi_{1}$ on a subset $\mathscr{D}_{1}=\mathscr{D}_{0}-\mathscr{B}_{1}$ of $\mathscr{D}_{0}$ as shown in Figure 2, where $\mathscr{B}_{1}$ is the hatched region. The field $\Phi_{1}$ is constructed on $(0, \infty) \times(1 / 2,1)$ in a manner analogous to that of the $C^{\infty}$ direction field $\Phi$ appearing in the proof of Proposition 1 so that if $f$ is constant on integral curves of $\Phi_{1}$ then $d f=0$ at $(0,1 / 2)$. Now note that the part of $\Phi_{1}$ on $(0, \infty) \times(1 / 2,1)$ and the part of $\Phi_{1}$ on $(0, \infty) \times(0,1 / 2)$ below the hatched region of Figure 2 are equivalent in an obvious manner to $\Phi_{0}$ on $\mathscr{D}_{0}$. Therefore, we can apply the same modification described above to each of these parts to obtain a field $\Phi_{2}$ on a domain $\mathscr{D}_{2}$ as shown in Figure 3. The only changes taking place occur for $x>1$. Now if $f$ is constant on integral curves of $\Phi_{2}$, then $d f=0$ on $\{(0,1 / 4),(0,1 / 2)$, $(0,3 / 4)\}$. We continue the iteration until we obtain a field $\Phi$ on a domain $\mathscr{D}$ which satisfies (i) and (ii). Clearly the resulting domain $\mathscr{D}$ is open, connected, and simply connected.

REMARK. Suppose the hatched regions in Figures 2 and 3 and in subsequent iterations are constructed so that their upper and lower boundary curves meet tangentially. In fact this is the way they appear in Figures 2 and 3. Then $\Phi$ extends continuously to the boundary of $\mathscr{D}$ by requiring, as apropriate, that $\Phi$ be horizontal or tangent to the boundary

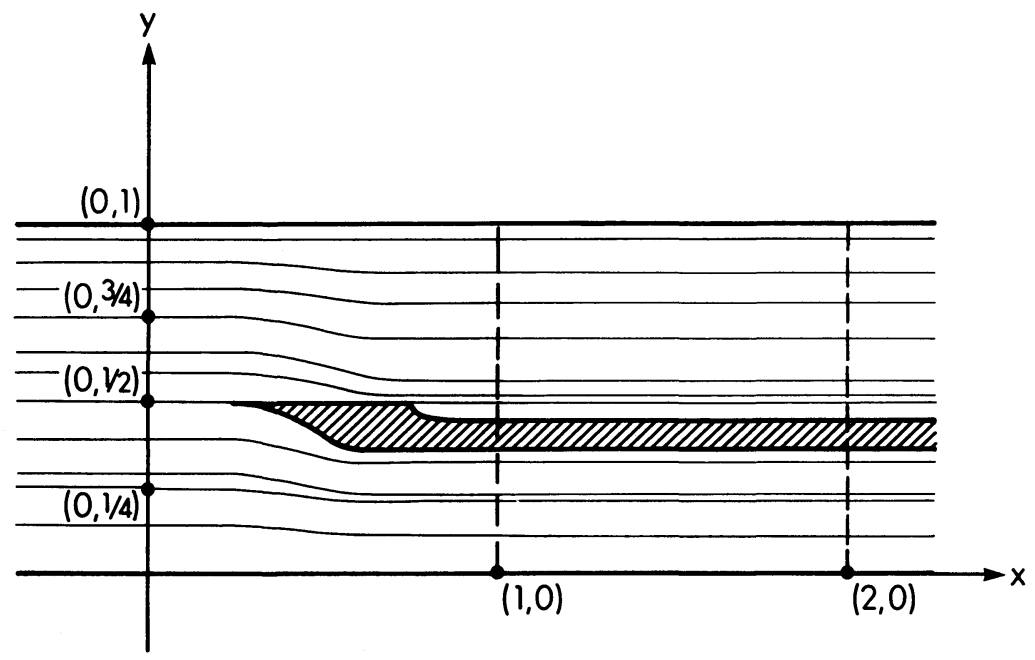

FIGURE 2 


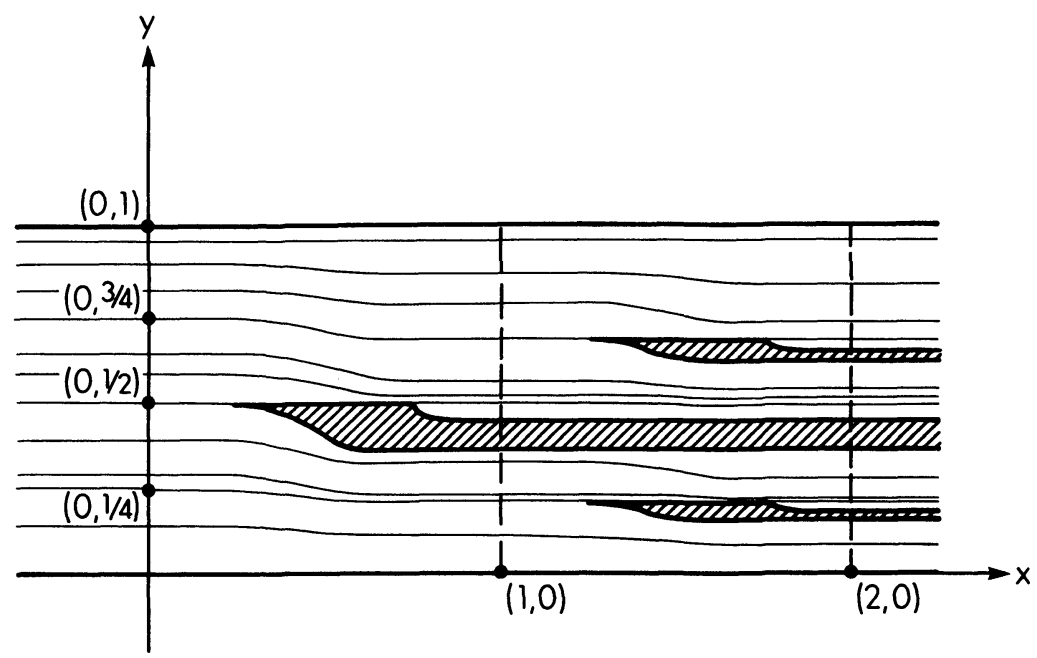

FIGURE 3

at each point of the boundary. In fact, for this extended $\Phi$ on $\overline{\mathscr{D}}$,

$$
\lim _{x \rightarrow \infty} \Phi(x, y)=\text { horizontal }
$$

because the modifications that occur further and further to the right in the construction of $\Phi$ are scaled down more and more in the vertical direction, but there is no scaling in the horizontal direction. Hence $\Phi$ extends to a continuous direction field on the closure of $\mathscr{D}$ in $\mathbf{R}^{2} \cup$ \{point at infinity\}.

COROLlARY. There is a first order linear homogeneous partial differential equation with $C^{\infty}$ coefficients defined on $\mathbf{R}^{2}$ containing an open subset $U$ such that any global $C^{1}$ solution of the p.d.e. is constant on $U$.

Proof. Let $\Phi$ be the direction field given in the proof of Theorem 1 . There is a nonvanishing $C^{\infty}$ vector field $X$ with $X(P) \in \Phi(P)$, for all $P$. Clearly $X u=0$ is the p.d.e. with the required properties.

2. Existence Theorems. We turn our attention to finding necessary and sufficient conditions on a $C^{r}$ direction field $\Phi$ in order that it possesses a $C^{r}$ first integral in the strong sense.

Definition 3. Give $\mathscr{F}$ the quotient topology induced from $\mathbf{R}^{2}$ and let $\pi: \mathbf{R}^{2} \rightarrow \mathscr{F}$ be the natural projection of $\mathbf{R}^{2}$ onto $\mathscr{F}$. Let $\mathscr{I}, \mathscr{J} \in \mathscr{F}$. We say that $\mathscr{I}$ is related to $\mathscr{J}$ if $U \cap V \neq \varnothing$ whenever $U$ and $V$ are open sets 
of $\mathscr{F}, \mathscr{I} \in U$, and $\mathscr{J} \in V$; i.e., $\mathscr{I}$ and $\mathscr{J}$ cannot be separated by open sets. Suppose that $\mathscr{I}$ is related to $\mathscr{J}$. This means that, given $P \in \mathscr{I}$ and $Q \in \mathscr{J}$, all integral curves of $\Phi$ that pass through a sufficiently small neighborhood of $P$ on a suitable side of $\mathscr{I}$ also pass through and cover an appropriate "half" of a certain neighborhood of $Q$. See the curves $u<-1$, $v=0$ and $u>1, v=0$ in Figure 1. Given transversals $\alpha$, resp. $\beta$, of $\mathscr{I}$, resp. $\mathscr{J}$, (properly oriented) such that $\alpha(0)=P$ and $\beta(0)=Q$, there clearly exists a unique continuous monotone function $\tau:[0, \varepsilon) \rightarrow[0, \varepsilon)$, for some $\varepsilon>0$, such that $\pi \circ \alpha=\pi \circ \beta \circ \tau$ on $[0, \varepsilon)$. We say the related $\mathscr{I}$ and $\mathscr{J}$ are $r$-compatible if $\tau$ is $C^{r}$ on $[0, \varepsilon)$, i.e., it is $C^{r}$-extendable to $(-\varepsilon, \varepsilon)$, and $\tau^{\prime}(0)>0$. The idea of $r$-compatibility is well-defined since the definition does not depend on the choice of the transversal curves, $\alpha$ and $\beta$; this follows from standard results from the theory of ordinary differential equations. If $\mathscr{I}=\mathscr{J}$ then, of course, $\mathscr{I}$ is related to $\mathscr{J}$, but $\mathscr{I}$ is also $r$-compatible with $\mathscr{J}$ again by standard results from the theory of ordinary differential equations. Finally, $\Phi$ is said to be $r$-normal if each related pair of members of $\mathscr{F}$ is $r$-compatible.

Obviously the direction fields constructed in the proofs of Proposition 1 and Theorem 1 are not even 1-normal.

TheOREM 2. Let $\Phi$ be a $C^{r}$ direction field on $\mathbf{R}^{2}$. A necessary and sufficient condition for $\Phi$ to possess a $C^{r}$ first integral in the strong sense is that $\Phi$ be r-normal.

Proof. Suppose $f$ is a $C^{r}$ first integral of $\Phi$ on $\mathbf{R}^{2}$ in the strong sense. Hence $f$ is constant on members of $\mathscr{F}$ and $d f$ does not vanish on $\mathbf{R}^{2}$. Assume $\mathscr{I}$ and $\mathscr{J}$ are distinct related members of $\mathscr{F}$. The function $\tau$, defined by relatedness, clearly satisfies the relation $f \circ \alpha=f \circ \beta \circ \tau$. Since $f \circ \alpha$ and $f \circ \beta$ have non-zero derivatives at 0 it follows that $\tau$ is $C^{r}$ and $\tau^{\prime}(0) \neq 0$. Therefore $\mathscr{I}$ and $\mathscr{J}$ are $r$-compatible. Consequently $\Phi$ is $r$-normal.

Now suppose $\Phi$ is $r$-normal. We define a flow-box at $P \in \mathbf{R}^{2}$ to be a diffeomorphism $\phi:[-1,1] \times[-1,1] \rightarrow \mathbf{R}^{2}$ with $\phi(0,0)=P$ such that $\phi([-1,1] \times\{\eta\})$ goes into an integral curve of $\Phi$ for $|\eta| \leq 1$. That $\phi$ is a diffeomorphism on a closed subset of $\mathbf{R}^{2}$ necessarily means that $\phi$ is extendable as a diffeomorphism to a neighborhood of $[-1,1] \times[-1,1]$ in $\mathbf{R}^{2}$. Flow-boxes exist for each $P$ in the plane. Let $\left\{S_{1}, S_{2}, \ldots\right\}$ be a sequence of flow-boxes whose interiors, $S_{i}^{0}, i=1,2, \ldots$, cover the plane. For any set $W$ in the plane, define the saturation $\tilde{W}$ of $W$ to be the union of the integral curves of $\Phi$ through $W$. It is well known that if $W$ is open and connected then so is $\tilde{W}$. 
On the interior of $\tilde{S}_{1}$ define $g_{1}: \tilde{S}_{1}^{0} \rightarrow \mathbf{R}$ by requiring that $g_{1}$ be constant on members of $\mathscr{F}$ and equal to $\eta$ (in the obvious sense) when restricted to $S_{1}^{0}$. This $g_{1}$ is $C^{r}$ and $d g_{1} \neq 0$ at any point. If $\tilde{S}_{1}^{0}=\mathbf{R}^{2}$, we are done. If not, we proceed as follows. For $i=1,2, \ldots$, let $h_{i}: \mathbf{R} \rightarrow \mathbf{R}$ be a $C^{\infty}$ diffeomorphism satisfying the following:

(i) $h_{i} \mid[-i+1, i-1]=$ identity,

(ii) $h_{i}$ (R) $\subset[-i, i]$.

Set $f_{1}=h_{1} \circ g_{1}$. Of course, $f_{1}$ is $C^{r}$ and $d f_{1} \neq 0$ at any point. Let $n_{2}$ be the smallest $n>1$ with $S_{n}^{0} \nsubseteq \tilde{S}_{1}^{0}$, but $S_{n}^{0} \cap \tilde{S}_{1}^{0} \neq \varnothing ; n_{2}$ exists by the connectedness of the plane. We claim $S_{n_{2}} \cap \tilde{S}_{1}^{0}$ is connected. If not, pick a point "between" two components, and consider the integral curve of $\Phi$ through it. It is an immediate consequence of Bendixon's Theorem [5, p. $219]$ that this curve goes to $\infty$ in both directions and separates the plane into two open subsets. Clearly $\tilde{S}_{1}^{0}$ intersects both parts, contradicting its connectedness. Now the $r$-normality of $\Phi$ allows us to extend $f_{1}$ to a $C^{r}$-function on $S_{n_{2}}$, constant on the integral curves of $\Phi$ and with differential nowhere 0 ; for $r<\infty$, this is easy, while for $r=\infty$ one may use the Borel Extension Lemma [2]. And this function extends by constancy on $\Phi$ integral curves to a function $g_{2}: \tilde{S}_{1}^{0} \cup \tilde{S}_{n_{2}}^{0} \rightarrow \mathbf{R}$. Then set $f_{2}=h_{2} \circ g_{2}$. Clearly $f_{2}$ is a first integral of $\Phi$ in the strong sense on $\tilde{S}_{1}^{0} \cup \tilde{S}_{n_{2}}^{0}$. Its domain, $\tilde{S}_{1}^{0} \cup \tilde{S}_{n_{2}}^{0}$, is again connected and its range being contained in $[-2,2]$ is not all of $\mathbf{R}$. Also $f_{2}$ agrees with $f_{1}$ on $\tilde{S}_{1}^{0}$, by the definition of $h_{2}$. Thus we may iterate this procedure, if necessary. Clearly this uses up all the $S_{i}$ by the connectedness of the plane.

The result of E. Kamke [3] and R. Finn [1] for the simply connected case follows easily from Theorem 2 .

COROllaRy 1. Let $\Phi$ be a $C^{r}$ direction field defined on a plane domain $\mathscr{E}$. Let $\mathscr{D}$ be a simply connected plane domain such that $\bar{D}$ is a compact subset of $\mathscr{E}$. Then $\Phi \mid \mathscr{D}$ has a $C^{r}$ first integral in the strong sense.

Proof. Suppose $\mathscr{I}_{1}$ and $\mathscr{I}_{2}$ are distinct related members of $\mathscr{F}$. Using standard results from the theory of o.d.e. and the fact that $\bar{D}$ is compact and contained in $\mathscr{E}$, we may show there exists a maximal integral curve $\mathscr{J}$ of $\Phi$ in $\mathscr{E}$ such that $\mathscr{I}_{n} \subset \mathscr{J}$, for $n=1,2$. Consequently $\mathscr{I}_{1}$ and $\mathscr{I}_{2}$ are $r$-compatible. Hence $\Phi \mid \mathscr{D}$ is $r$-normal and the corollary follows from Theorem 2.

Suppose that whenever the maximal integral curves $\mathscr{I}$ and $\mathscr{J}$ of $\Phi$ are related it follows that $\mathscr{I}=\mathscr{J}$. This implication is clearly equivalent to the statement that $\mathscr{F}$ with its quotient topology is a Hausdorff space. 
Also, it is an immediate consequence of this implication that $\Phi$ is $r$-normal. These observations yield the next corollary.

Corollary 2. Let $\Phi$ be a $C^{r}$ direction field on $\mathbf{R}^{2}$. Suppose the space of maximal integral curves, $\mathscr{F}$, of $\Phi$ is a Hausdorff space. Then $\Phi$ has a $C^{r}$ first integral in the strong sense.

REMARK 1. By using an induction proof similar to that employed in the proof of Theorem 2 and W. Kaplan's Theorem 11 from [4] in place of Bendixson's Theorem, a shorter and more transparent proof may be given of W. Kaplan's result that regular curve-families possess "continuous first integrals", Theorem 42 of [4]. However, we lose all the results on the structure of such curve-families that W. Kaplan obtained in getting his result.

REMARK 2. Suppose that $\mathscr{F}$ contains a finite number of integral curves that are related and not $r$-compatible. Then it seems reasonable to conjecture that $\Phi$ should possess a $C^{r}$ first integral in the weak sense. The requirement that the number of such curves be finite is necessary; this is a consequence of the example appearing in the proof of Theorem 1.

We would like to strengthen the result of Theorem 2 by showing that if $\Phi$ is $r$-normal then $\Phi$ can be "uniformized." Precisely, we will show that there exists a $C^{r}$ local diffeomorphism $\mu: \mathbf{R}^{2} \rightarrow \mathbf{R}^{2}$ such that $\Phi$ is mapped under $\mu$ into the (constant) vertical direction field. First we need the following lemma.

LEMMA. Let $f: \mathbf{R}^{2} \rightarrow \mathbf{R}$ be a $C^{r}$ function whose differential, $d f$, never vanishes. Then there exists a $C^{r}$ function $g: \mathbf{R}^{2} \rightarrow \mathbf{R}$ such that $d f \wedge d g>0$.

Proof. We will prove this lemma under the assumption that $r=\infty$. The proof of this result for $r<\infty$ is somewhat easier and ought to be suggested by the proof for the case $r=\infty$.

Throughout this proof let $b: \mathbf{R} \rightarrow \mathbf{R}$ be a $C^{\infty}$ function satisfying the following conditions:

(1) $b(t)=0$, for $|t| \geq 1$.

(2) $0<b(t) \leq 1$, for $|t|<1$.

Also suppose that $l: \mathbf{R} \rightarrow \mathbf{R}$ is a fixed $C^{\infty}$ function satisfying the following conditions:

(1) $l(t)=0$, for $t \leq-1$.

(2) $l(t)=1$, for $t \geq 1$.

(3) $l^{\prime}(t)>0$, for $|t|<1$. 
For each point $P \in \mathbf{R}^{2}$ there exists an open neighborhood $V$ of $P$ such that $\bar{V}$ is compact; in addition, there exists a coordinate system $(x, y)$ : $V \rightarrow(-2,2) \times(-2,2)$ such that
(a) $x=\mu(f-a)$, for reals $a$ and $\mu$ with $\mu>0$, and
(b) $d x \wedge d y>0$.

Note that $x$ is defined on all of $\mathbf{R}^{2}$ and that $d f \wedge d y>0$. Hence there exists a countable collection of such $V$ 's which we denote by $\left\{V_{k} \mid k \in \mathbf{Z}^{+}\right\}$ with associated coordinates $x_{k}=\mu_{k}\left(f-a_{k}\right)$ and $y_{k}$ such that $\left\{U_{k} \mid k \in\right.$ $\left.\mathbf{Z}^{+}\right\}$covers $\mathbf{R}^{2}$ where $U_{k}=\left(x_{k}, y_{k}\right)^{-1}[(-1,1) \times(-1,1)]$. For each $k \in \mathbf{Z}^{+}$ we define a function $g_{k}: \mathbf{R}^{2} \rightarrow \mathbf{R}$ as follows: On $V_{k}$ let $g_{k}=b\left(x_{k}\right) l\left(y_{k}\right)$. On $\left\{P \in \mathbf{R}^{2}|| x_{k}(P) \mid>1\right\}$ let $g_{k}=0$. Let $\mathscr{E}_{k}$ be the component of $\left\{P \in \mathbf{R}^{2}|| x_{k}(P) \mid<2\right\}$ that contains $U_{k}$. The curves $y_{k}= \pm 1$ separate $\mathscr{E}_{k}$ into three connected components; let $\mathscr{A}_{k}$, resp. $\mathscr{B}_{k}$, be that component of $\mathscr{E}_{k}-\left\{P \in \mathbf{R}^{2} \mid y_{k}(P)= \pm 1\right\}$ containing points where $y_{k}>1$, resp. $y_{k}<$ -1. Let $g_{k}=b\left(x_{k}\right)$ on $\mathscr{A}_{k}$ and suppose $g_{k}=0$ on $\mathscr{B}_{k}$ and on $\{P \in$ $\left.\mathbf{R}^{2}|| x_{k}(P) \mid<2\right\}-\mathscr{E}_{k}$. The function $g_{k}$ so defined is indeed a well-defined $C^{\infty}$ function on $\mathscr{D}$. Note that $\left|g_{k}\right| \leq 1$ on $\mathbf{R}^{2}$. Also $d g_{k} \equiv 0$ $(\bmod d f)$ on $\mathbf{R}^{2}-U_{k}$ since $d x_{k}=\mu_{k} d f$ and $d g_{k} \equiv b\left(x_{k}\right) l^{\prime}\left(y_{k}\right) d y$ $(\bmod d f)$ on $U_{k}$. Hence $d f \wedge d g_{k} \geq 0$ and, in fact, $d f \wedge d g_{k}>0$ on $U_{k}$, for $k \in \mathbf{Z}^{+}$.

Introduce a metric $\langle$,$\rangle on \mathbf{R}^{2}$ by setting

$$
\langle,\rangle=(d f)^{2}+\omega^{2}
$$

where $\omega$ is a 1 -form on $\mathbf{R}^{2}$ such that $d f \wedge \omega>0$. If $\Psi$ is a symmetric multilinear form on $\mathbf{R}^{2}$ let $\|\Psi\|: \mathbf{R}^{2} \rightarrow \mathbf{R}$ denote the norm of $\Psi$ with respect to $\langle$,$\rangle . For j>0$, let $d^{(j)} g_{k}$ denote the $j$ th order differential of $g_{k}$; of course, we write $d g_{k}$ for $d^{(1)} g_{k}$. Off $\mathscr{A}_{k} \cup U_{k}$ necessarily $d^{(j)} g_{k}=0$ for $j>0$. On $\mathscr{A}_{k}, d^{(j)} g_{k}=b^{(j)}\left(x_{k}\right) \mu_{k}^{j}(d f)^{j}$, where $(d f)^{j}$ denotes the $j$ th symmetric product of $d f$ with itself. Consequently, there exists a constant $M_{k}^{\prime}$ such that $\left\|d^{(j)} g_{k}\right\|<M_{k}^{\prime}$ on $A_{k}$, for $1 \leq j \leq k$. Since $U_{k}$ has compact closure there exists a constant $M_{k}^{\prime \prime}$ such that $\left\|d^{(j)} g_{k}\right\|<M_{k}^{\prime \prime}$ on $U_{k}$ for $1 \leq j \leq k$. Let $M_{k}=\max \left\{1, M_{k}^{\prime}, M_{k}^{\prime \prime}\right\}$. Set $g=\sum_{k=1}^{\infty} 2^{-k} M_{k}^{-1} g_{k}$; since $\left|2^{-k} M_{k}^{-1} g_{k}\right| \leq 2^{-k}$, for all $k$, the series converges uniformly to $g$. Moreover, $\sum_{k=1}^{\infty} 2^{-k} M_{k}^{-1} d^{(j)} g_{k}$ converges uniformly, for $j>0$, since $\left\|2^{-k} M_{k}^{-1} d^{(j)} g_{k}\right\| \leq 2^{-k}$ for $k \geq j$. Hence $g$ is $C^{\infty}$ on $\mathbf{R}^{2}$. In particular, $d g=\sum_{k=1}^{\infty} 2^{-k} M_{k}^{-1} d g_{k}$; thus $d f \wedge d g=\sum_{k=1}^{\infty} 2^{-k} M_{k}^{-1} d f \wedge d g_{k}>0$ since, as noted above, $d f \wedge d g_{k} \geq 0$, $d f \wedge d g_{k}>0$ on $U_{k}$, for all $k$, and $\left\{U_{k}\right\}$ covers $\mathbf{R}^{2}$.

REMARK. Note that the lemma still holds if we replace $\mathbf{R}^{2}$ by a domain which is not simply connected. 
We remind the reader that a $C^{r}$ local diffeomorphism $\mu: \mathbf{R}^{2} \rightarrow \mathbf{R}^{2}$ is a $C^{r}$ map whose differential, $d \mu$, is an isomorphism at each point of $\mathbf{R}^{2}$. Hence locally $\mu$ has a $C^{r}$ inverse. If $\Phi$ is a direction field on $\mathbf{R}^{2}$, we can use $d \mu$ to assign to $\Phi(P)$ the direction $d \mu(\Phi(P))$ at $\mu(P) \in \mu\left(\mathbf{R}^{2}\right)$, for each $P \in \mathbf{R}^{2}$. In general this procedure will not define a direction field on $\mu\left(\mathbf{R}^{2}\right)$, but if it does the resulting direction field is denoted by $d \mu(\Phi)$.

THeOREM 3. Let $\Phi$ be a $C^{r}$ direction field on $\mathbf{R}^{2}$. Then $\Phi$ is $r$-normal if and only if there exists a $C^{r}$ local diffeomorphism $\mu: \mathbf{R}^{2} \rightarrow \mathbf{R}^{2}$ such that $d \mu(\Phi)$ is the (constant) vertical direction field.

REMARK. The condition that $d \mu(\Phi)$ be the vertical direction field is equivalent to asserting that $\mu$ maps members of $\mathscr{F}$ into vertical line segments.

Proof. The necessity of $\Phi$ being $r$-normal is obvious. On the other hand, if $\Phi$ is $r$-normal, then $\Phi$ possesses a $C^{r}$ first integral $f: \mathbf{R}^{2} \rightarrow \mathbf{R}$ in the strong sense. Then the lemma assures the existence of a $C^{r}$ function $g$ : $\mathbf{R}^{2} \rightarrow \mathbf{R}$ such that $d f \wedge d g>0$. Set $\mu=(f, g)$. Clearly $\mu$ is a $C^{r}$ local diffeomorphism since $d f \wedge d g>0$. It is equally clear that $d \mu(\Phi)$ is vertical since $d f(\Phi)=0$.

One cannot help wondering whether or not it is possible to insist that the mapping $\mu$ in Theorem 3 be a diffeomorphism rather than a local diffeomorphism. However, the following example with $\mathbf{R}^{2}$ again replaced by a suitable domain $\mathscr{D}$ shows that Theorem 3 is the best one can hope for. Consider the normal direction field $\Phi$ whose maximal integral curves are indicated in Figure 4a by heavy and light curves. Suppose there exists a diffeomorphism $\mu: \mathscr{D} \rightarrow \mu(\mathscr{D}) \subset \mathbf{R}^{2}$ such that $d \mu(\Phi)$ is vertical. The members of $\mathscr{F}$ indicated by the heavier lines and labeled $\mathscr{I}_{1}, \mathscr{I}_{2}$, and $\mathscr{I}_{3}$ in Figure 4a must be mapped by $\mu$ to vertical line segments all of which lie in the same line $\ell$ of $\mathbf{R}^{2}$. The line $\ell$ is indicated in Figure $4 \mathrm{~b}$ by dots; the images of $\mathscr{I}_{1}, \mathscr{I}_{2}$, and $\mathscr{I}_{3}$ under $\mu$ are indicated by heavy vertical line segments and are labeled simply by 1,2 , and 3, respectively. The part of $\mathscr{D}$ that lies on and above $\mathscr{I}_{3}$ must be mapped by $\mu$ to the sort of region depicted in Figure $4 \mathrm{~b}$. The light vertical lines in Figure $4 \mathrm{~b}$ indicate the images of members of $\mathscr{F}$ under $\mu$. Necessarily the part of $\mathscr{D}$ that lies below $\mathscr{I}_{3}$ must be mapped by $\mu$ to a region of the sort indicated by the diagonal hatching in Figure 4c. This contradicts the assumption that $\mu$ is a diffeomorphism. Hence it is impossible to find a diffeomorphism $\mu$ : $\mathscr{D} \rightarrow \mathbf{R}^{2}$ such that $d \mu(\Phi)$ is vertical. There are other examples in the 


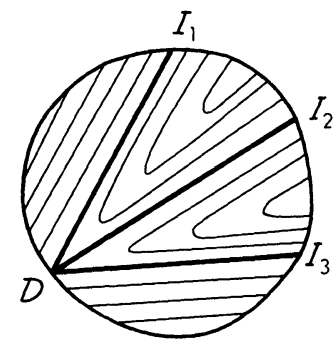

FigURE 4a

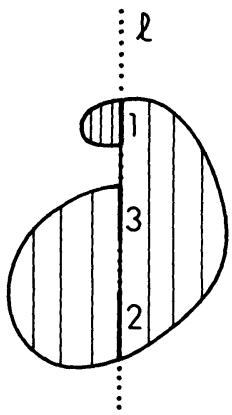

FigURE $4 b$

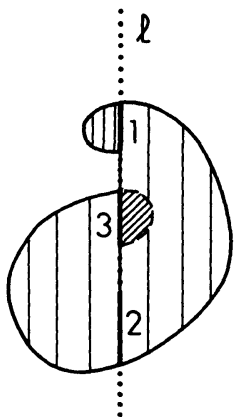

FIGURE 4c

literature of direction fields $\Phi$ defined on simply connected plane domains such that there exist no diffeomorphisms $\mu$ which "uniformize" $\Phi$. See, for example, Finn [1, Figure 3].

Suppose $\Phi$ and $\Psi$ are $C^{r}$ direction fields on $\mathbf{R}^{2}$. We say that $\Phi$ and $\Psi$ are independent if $\Phi(P) \neq \Psi(P)$, for all $P \in \mathbf{R}^{2}$.

Theorem 4. Suppose $\Phi$ and $\Psi$ are independent $C^{r}$ direction fields on $\mathbf{R}^{2}$. Both $\Phi$ and $\Psi$ are r-normal if and only if there exists a local diffeomorphism $\mu: \mathbf{R}^{2} \rightarrow \mathbf{R}^{2}$ such that $d \mu(\Phi)$ is the vertical direction field and $d \mu(\Psi)$ is the horizontal directional field.

Proof. Clearly $\Phi$ and $\Psi$ must be $r$-normal if $d \mu(\Phi)$ and $d \mu(\Psi)$ are constant direction fields for some local diffeomorphism $\mu$. On the other hand, if $\Phi$ and $\Psi$ are $r$-normal then Theorem 2 implies there exists $C^{r}$ first integrals $f$ and $g$ for $\Phi$ and $\Psi$, resp., in the strong sense. Since $\Phi$ and $\Psi$ are independent, it follows that $d f \wedge d g$ never vanishes. Setting $\mu=$ $(f, g)$ we obtain the desired $C^{r}$ local diffeomorphism.

3. Applications. We shall apply our results to the theory of differentiable manifolds and to the normalization of second order linear partial differential equations defined on simply connected plane domains.

Suppose $M$ is a $C^{\infty}$ differentiable manifold and $\phi: \mathbf{R}^{2} \rightarrow M$ is a $C^{r+1}$ mapping. We say $\phi$ is a rank 1 mapping if the differential of $\phi$ at $P$, denoted $d \phi_{\mid P}$, has rank 1 for each $P \in \mathbf{R}^{2}$. If $\phi$ is a rank 1 mapping, then a $C^{r}$ direction field $\Phi$ may be defined on $\mathbf{R}^{2}$ by setting

$$
\Phi(P)=\operatorname{ker} d \phi_{\mid P},
$$

for all $P \in \mathbf{R}^{2}$. We will also denote this field $\Phi$ by $\operatorname{ker} d \phi$. 
THEOREM 4. Suppose $\phi: \mathbf{R}^{2} \rightarrow M$ is a $C^{r+1}$ mapping from $\mathbf{R}^{2}$ into a differentiable manifold $M$. If $\phi$ is a rank 1 mapping then there exists a $C^{r}$ local diffeomorphism $\mu: \mathbf{R}^{2} \rightarrow \mathbf{R}^{2}$ which maps $\operatorname{ker} d \phi$ onto the vertical direction field on $\mu(\mathscr{D})$.

Proof. By Theorem 3, it is enough to show that $\Phi=\operatorname{ker} d \phi$ is $r$-normal. Thus let $\mathscr{I}$ and $\mathscr{J}$ be related members of $\mathscr{F}$. Let $\alpha, \beta$ : $(-\varepsilon, \varepsilon) \rightarrow \mathbf{R}^{2}$ be curves having all the properties given to the curves with the same names that appear in Definition 3. Then $\phi \circ \alpha, \phi \circ \beta:(-\varepsilon, \varepsilon) \rightarrow \mu$ are $C^{r+1}$ curves in $M$ with nonvanishing velocities. Also $\phi \circ \alpha([0, \varepsilon)) \cap$ $\phi \circ \beta([0, \varepsilon)) \neq \varnothing$. By introducing a Riemannian metric on $M$ we can reparametrize $\alpha$ and $\beta$, resp., with respect to the arc length along $\phi \circ \alpha$ and $\phi \circ \beta$, resp., measured from $\phi \circ \alpha(0)=\phi \circ \beta(0)$ in the direction of $\phi \circ \alpha([0, \varepsilon)) \cap \phi \circ \beta([0, \varepsilon))$. With respect to these new arc length parameters, the function $\tau:[0, \varepsilon) \rightarrow[0, \varepsilon)$ introduced in Definition 3 is the identity function. Hence $\tau$ is $C^{r}$ on $[0, \varepsilon)$ and $\tau^{\prime}(0)=1>0$. Thus $\mathscr{I}$ and $\mathscr{J}$ are $r$-compatible. This proves $\operatorname{ker} d \phi$ is $r$-normal.

Remark. We may view $\mu: \mathbf{R}^{2} \rightarrow \mathbf{R}^{2}$ as a global "coordinate system" on $\mathbf{R}^{2}$ relative to which the mapping $\phi$ has a particularly simple form since $\phi$ depends on just one "coordinate" of this "coordinate system." As an application of the sort of global results one may obtain from this kind of "coordinate system" see Theorem 1 in [7].

We now turn our attention to normalizing second order partial differential equations of the form

$$
a(x, y) u_{x x}+2 b(x, y) u_{x y}+c(x, y) u_{y y}+d\left(x, y, u, u_{x}, u_{y}\right)=0
$$

defined in a simply connected plane domain $\mathscr{D}$. We assume that $a^{2}+b^{2}$ $+c^{2} \neq 0$ and $a, b, c$ are of class $C^{2}$ throughout $\mathscr{D}$. If (1) is hyperbolic on $\mathscr{D}$ then it determines a pair of independent $C^{2}$ direction fields on $\mathscr{D}$, the characteristic direction fields, which we denote by $\Phi$ and $\Psi$. If both $\Phi$ and $\Psi$ are 2-normal in $\mathscr{D}$ then Theorem 4 asserts the existence of a $C^{2}$ local diffeomorphism $\mu: \mathscr{D} \rightarrow \mathbf{R}^{2}$ which maps the characteristic curves of (1) into vertical and horizontal lines in $\mathbf{R}^{2}$. Suppose $\mu=(\xi, \eta)$. Since $\mu$ is a $C^{2}$ local diffeomorphism, locally $(\xi, \eta)$ is a $C^{2}$ coordinate system in $\mathscr{D}$. Hence $\partial / \partial \xi, \partial / \partial \eta, \partial^{2} / \partial \xi^{2}, \partial^{2} / \partial \xi \partial \eta$, and $\partial^{2} / \partial \eta^{2}$ make sense throughout $\mathscr{D}$. Hence with respect to the "coordinate system" $(\xi, \eta)$ the equation (1) takes on its normal form

$$
u_{\xi \eta}+\delta\left(\xi, \eta, u, u_{\xi}, u_{\eta}\right)=0
$$


on $\mathscr{D}$, where admittedly $u$ and $\delta$ are multiple-valued functions of $\xi$ and $\eta$. We may view the local diffeomorphism $\mu$ as changing (1) on $\mathscr{D}$ into (2) on the non-schlicht domain $\mu(\mathscr{D})$, i.e., a self-overlapping domain like that depicted in Figure 4c. Domains of influence and determinacy are still meaningful for non-schlicht domains; one just must be careful not to jump from one "level" to another "level" of such a domain. Hence $\mu(\mathscr{D})$ is appropriate for the study of global solutions of (1).

If (1) was parabolic on $\mathscr{D}$, we should only have to assume that its field of characteristic directions was 2-normal in order to be able to apply Theorem 3 and then make remarks similar to the above about (1) in this case.

\section{REFERENCES}

[1] R. Finn, On the uniformization of plane direction fields, and of second-order partial differential operations, Proc. Camb. Phil. Soc., 73 (1973), 87-109.

[2] M. Golubitsky and V. Guillemin, Stable Mappings and Their Singularities, SpringerVerlag, New York, Heidelberg, Berlin, 1973.

[3] E. Kamke, Über die partielle Differentialgleichung $f(x, y) \partial z / \partial x+g(x, y) \partial z / \partial y=$ $h(x, y)$. II, Math. Z., 42 (1936), 287-300.

[4] W. Kaplan, Regular curve-families filling the plane, I, Duke Math. J., 7 (1940), 154-185.

[5] S. Lefschetz, Differential Equations: Geometric Theory, Interscience Publishers, Inc., New York, 1957.

[6] T. Wazewski, Sur un problème de caractère intégral relatif à l'équation $\partial z / \partial x+$ $Q(x, y) \partial z / \partial y=0$, Mathematica Cluj, 8 (1934), 103-116.

[7] J. Weiner, The Gauss map for surfaces-Part I. The affine case, Trans. Amer. Math. Soc., 293 (1986), 431-446.

Received August 2, 1983.

UNIVERSITY OF HAWAII AT MANOA

2565 THE MALL

HONOLULU, HI 96822 


\section{PACIFIC JOURNAL OF MATHEMATICS \\ EDITORS}

V. S. VARADARAJAN

(Managing Editor)

University of California

Los Angeles, CA 90024

Herbert Clemens

University of Utah

Salt Lake City, UT 84112

R. FINN

Stanford University

Stanford, CA 94305
HERMANN FLASCHKA

University of Arizona

Tucson, AZ 85721

RAMESH A. GANGOLLI

University of Washington Seattle, WA 98195

VAUGHAN F. R. JONES

University of California

Berkeley, CA 94720
ROBION KIRBY

University of California

Berkeley, CA 94720

C. C. MOORE

University of California

Berkeley, CA 94720

HAROLD STARK

University of California, San Diego

La Jolla, CA 92093

\section{ASSOCIATE EDITORS}
R. ARENS
E. F. BECKENBACH
B. H. NEUMANN
F. WOLF
K. YOSHIDA
(1906-1982)

\section{SUPPORTING INSTITUTIONS}

UNIVERSITY OF ARIZONA

UNIVERSITY OF BRITISH COLUMBIA

CALIFORNIA INSTITUTE OF TECHNOLOGY

UNIVERSITY OF CALIFORNIA

MONTANA STATE UNIVERSITY

UNIVERSITY OF NEVADA, RENO

NEW MEXICO STATE UNIVERSITY

OREGON STATE UNIVERSITY
UNIVERSITY OF OREGON UNIVERSITY OF SOUTHERN CALIFORNIA

STANFORD UNIVERSITY

UNIVERSITY OF HAWAII

UNIVERSITY OF TOKYO

UNIVERSITY OF UTAH

WASHINGTON STATE UNIVERSITY

UNIVERSITY OF WASHINGTON 


\section{Pacific Journal of Mathematics \\ Vol. 132, No. $1 \quad$ January, 1988}

Scott W. Brown, Full analytic subspaces for contractions with rich spectrum ...1

Robert Main Burton, Jr. and Tae-Sung Kim, An invariance principle for

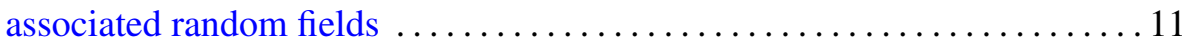

Ana M. Viola-Prioli and Jorge Viola-Prioli, Rings whose kernel functors

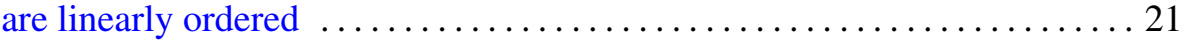

David E. Handelman, Representing polynomials by positive linear

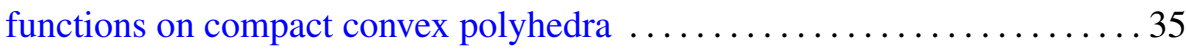

Patrick Keef, On the Tor functor and some classes of abelian groups . .....66

Dennis R. Malm, Simplicity of partial and Schmidt differential operator

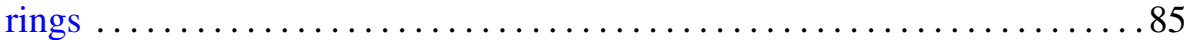

José M. Montesinos and Carmen Safont, On the Birman invariants of

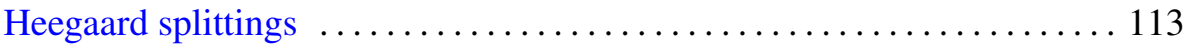

N. P. Mukherjee and Prabir Bhattacharya, The normal index of a finite group

Mario Petrich and Norman R. Reilly, Semigroups generated by certain operators on varieties of completely regular semigroups

Robert Tijdeman and Lian Xiang Wang, Sums of products of powers of given prime numbers

Joel Larry Weiner, First integrals for a direction field on a simply connected plane domain 